\title{
Resonant uv pump-probe spectroscopy of dipicolinic acid via impulsive excitation
}

\author{
Robert K. Murawski \\ Institute for Quantum Studies and Department of Physics, Texas A\&M University, College Station, Texas 77843-4242, USA \\ and Department of Physics, Drew University, Madison, New Jersey 07940, USA \\ Yuri V. Rostovtsev and Zoe-Elizabeth Sariyanni \\ Institute for Quantum Studies and Department of Physics, Texas A\&M University, College Station, Texas 77843-4242, USA \\ Vladimir A. Sautenkov \\ Institute for Quantum Studies and Department of Physics, Texas A\&M University, College Station, Texas 77843-4242, USA \\ and P. N. Lebedev Institute of Physics, 53 Leninsky prospect, Moscow 119991, Russia
}

Sterling Backus, Daisy Raymondson, Henry C. Kapteyn, and Margaret M. Murnane

Department of Physics and JILA, University of Colorado, Boulder, Colorado 80309-0440, USA

\author{
Marlan O. Scully \\ Institute for Quantum Studies and Department of Physics, Texas A\&M University, College Station, Texas 77843-4242, USA \\ and Applied Physics and Materials Science Group, Engineering Quadrangle, Princeton University, Princeton, New Jersey 08544, USA
}

(Received 6 November 2007; published 4 February 2008)

\begin{abstract}
We present experimental evidence of coherent wave packet motion in dipicolinic acid $\left(\mathrm{C}_{7} \mathrm{H}_{5} \mathrm{NO}_{4}\right)$ which is an important marker molecule for bacterial spores. Resonant impulsive excitation is achieved by applying a uv pump pulse $(267 \mathrm{~nm}, 16 \mathrm{fs})$ which has a duration that is shorter than the vibrational period of the molecules. The resulting dynamics is then probed with a weaker pulse of the same width and frequency. Evidence of the important "fingerprint" region for this molecule (between $1000 \mathrm{~cm}^{-1}$ and $1500 \mathrm{~cm}^{-1}$ ) is found in the transient absorption of the probe. We present simulations of the pump-probe experiment, based on the Liouville equation for the density matrix, and predict the optimal pulse width and detuning.
\end{abstract}

DOI: 10.1103/PhysRevA.77.023403

PACS number(s): 37.10.Jk, 42.65.Dr, 42.50.Hz

\section{INTRODUCTION}

Many optical spectroscopic techniques, including Raman scattering, have been successfully used in chemistry and biology [1]. The advent of femtosecond laser systems has opened new doors of research such as the pioneering work of Zewail and co-workers on ultrafast dynamics of molecules and fragmentation control $[2,3]$. The techniques of Raman scattering and ultrashort pulses have been combined into useful methods for studying molecules. One such method is impulsive stimulated raman scattering (ISRS) in which the laser pulse duration is shorter than the vibrational period of the molecule $[4,5]$.

The recent success of designing laser systems which produce ultrashort electronically resonant pulses $[6,7]$ (shorter than the vibrational period of molecular oscillation, and at short wavelengths that are resonant with electronic excited states in a wide variety of molecular systems) opens a new way to prepare molecular coherence. In the resonant version of ISRS (RISRS) [8-11], an electronically resonant broadband pulse is used to create molecular coherence in the ground state. This molecular coherence can then be probed by another ultrashort pulse. This allows one to directly observe molecular oscillations of biomolecules by monitoring the transient absorption of the probe in a pump-probe setup.

Recently, another scheme has been proposed to enhance the Raman signal by orders of magnitude. In the femtosecond adaptive spectroscopic technique for coherent antiStokes Raman scattering (FAST CARS), the quantum coher- ence between the vibrational states is maximized before probing the atoms or molecules by applying adaptively shaped laser pulses $[12,13]$.

Impulsive excitation provides some advantages due to its simplicity, using only one strong pulse resonant with the HOMO-LUMO electronic transition. In this paper, we present an experimental observation of ground state vibrational wave packet motion in dipicolinic acid (DPA), which is a marker for bacterial spores such as those formed by Bacillus anthracis which causes the anthrax disease [14]. A short ( $\approx 16 \mathrm{fs}$ ) uv pump pulse is used to excite some population from the ground state to the first excited electronic state. The bandwidth of the pump pulse is broad enough to excite several molecular vibrations. The delayed probe pulse interacts with the molecules which have been vibrationally excited and consequently the transmission of the probe will be modulated by the vibrational frequencies.

Shown in Fig. 1(a) is an energy surface schematic showing the process of the creation of a vibrational ground state wave packet via optically pumping with a short uv pulse $[15,16]$. The resonant pulse drives a portion of the stationary ground state wavepacket to the first excited electronic state. Here the wave packet is nonstationary and moves classically along the excited state surface. A portion of the same pulse can drive the wave packet back down coherently and now is at a nonstationary point. This wave packet will move in the ground state potential energy surface with a characteristic frequency given by the curvature of the well. Direct observation of molecular vibrations of biomolecules presents a 


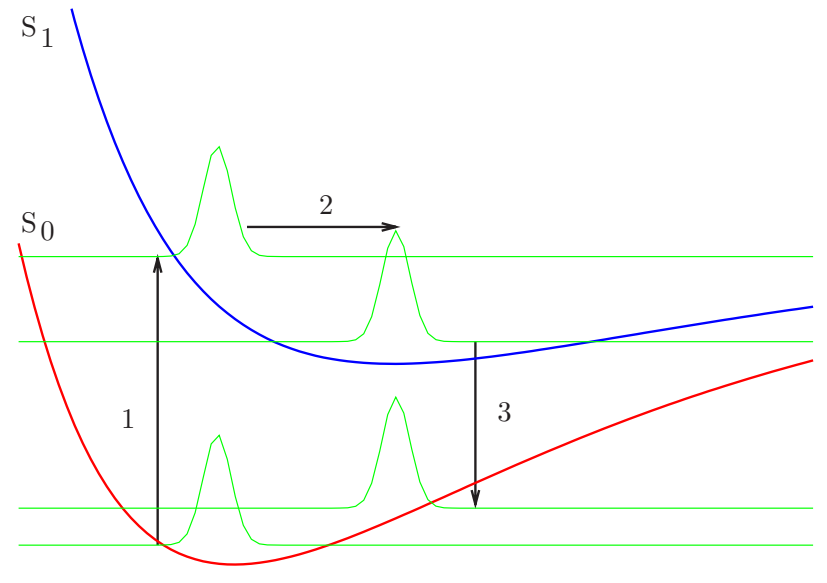

(a)Wavepacket motion

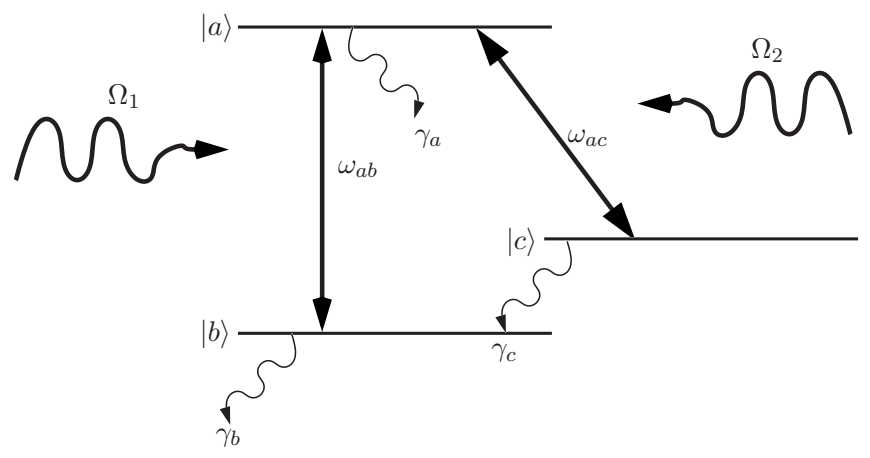

(b)Energy level diagram

FIG. 1. (Color online) (a) Schematic of the creation of the wave packet dynamics. Step 1: A portion of the stationary ground state wave packet is promoted to the excited state energy surface by means of a uv pump pulse. Step 2: The wave packet moves along the excited state energy surface. Step 3: The same uv pulse drives the excited state wave packet back down to the ground state energy surface. Now it is no longer at a stationary point but rather oscillates in the ground state, i.e., a ground state vibrational wave packet is created. (b) Simplified three-level scheme for the molecule. Here $\Omega_{1}$ and $\Omega_{2}$ are the amplitudes of the driving lasers in terms of their Rabi frequency, $\omega_{i j}$ is the energy difference between levels $i$ and $j$, and $\gamma_{i}$ is the dephasing rate for level $i$.

unique challenge because of the typically high vibrational frequencies $\sim 1000 \mathrm{~cm}^{-1}$ and thus requires a very different experimental parameters compared with the one used for $\mathrm{I}_{2}$ or $\mathrm{Na}_{2}$ where the vibrational frequencies are of the order of $30 \mathrm{~cm}^{-1}$ (period is $1 \mathrm{ps}$ ) $[17,18]$. Therefore, femtosecond lasers which provide ultrashort pulses are absolutely essential for biomolecular spectroscopy.

Additionally, we present simulations of the resonant impulsive technique based on the time evolution of the density matrix equations [20]. We have performed simulations, using parameters from the experiment and for DPA, which reveal molecular oscillations at $\sim 1000 \mathrm{~cm}^{-1}$ which is what we observed in the experiment. We have plans for more detailed simulations which will include propagation (see [21] for details).

\section{EXPERIMENTAL RESULTS}

A key challenge in this experiment was the fact that very short-duration pulses in the deep-uv are required for impulsive excitation. However, dispersion of ultrashort optical pulses is so severe in this region of the spectrum that few experiments of molecular dynamics in solution have been successfully implemented at a time resolution of less than 100 fs [22]. Furthermore, the very large values of high-order dispersion in this spectral region make conventional dispersion compensation extremely challenging. For example, the dispersion of a prism pair becomes pathological in the uv, while the efficiency of a diffraction grating compressor is very low. Thus, in this experiment, we avoid dispersion compensation by (1) completely avoiding transmissive optics in the beam path up until the sample, (2) using an ultrathin sample cell, and (3) keeping the total air path for the uv small, since even $\leqslant 1$ meter path length in air can substantially broaden the duration of the uv pulse in this range. This setup is implemented by using third harmonic generation from simply focusing the light from a Ti:sapphire laser amplifier system in air, creating a spark [23]. This light is generated through a $\chi^{(3)}$ interaction with the air, and the generation of a plasma defocuses the light to break the focal symmetry to avoid cancellation of the signal between the parts generated in the converging and diverging focuses [24].

We have used a Ti:sapphire amplifier which produces $22 \mathrm{fs}$ pulses at $800 \mathrm{~nm}$ with a $2 \mathrm{kHz}$ repetition rate and an average power of $2 \mathrm{~W}$. Following the method presented elsewhere [7], we generate a $16 \mathrm{fs}$ uv pulse $(267 \mathrm{~nm})$ by focusing the output of the amplifier in air which generates an air plasma. The air plasma is a nonlinear medium which gives the third harmonic of $800 \mathrm{~nm}$ and coincides with the absorption band of DPA. The uv pulses ( $1 \mu \mathrm{J}, 16 \mathrm{fs})$ are shorter then the IR pulses ( $1 \mathrm{~mJ}, 22 \mathrm{fs})$ but not as short as is optimally possible through a third harmonic process. Shown in Fig. 2(a) are the schematics of the experimental setup. The uv pulses are collimated and sent to a split focusing dielectric mirror. One part of the split mirror is mounted on a translation stage to control the delay time of the pump pulses. The pump and probe beams are focused onto the sample. The sample was DPA (Sigma Aldrich) dissolved in pure ethanol until saturated. The transmission through the solution was measured with a spectrophotometer and found to be 0.5 at $267 \mathrm{~nm}$. The solution is pumped through a flow cell, via a peristaltic pump, with a window thickness of 100 microns and solution thickness of 300 microns. To take data, we measured the probe with a fast photodiode. The photodiode signal was sent to a lock-amplifier and the pump was optically chopped at a rate of $1 \mathrm{kHz}$.

The data presented in Fig. 2(b) is lock-in signal versus pump delay. Variation of the transmission of the probe pulses versus delay time (position of translation stage) was recorded. Signals are different for pure ethanol and DPA solution dissolved in ethanol. There is evidence of oscillations in the DPA solution curve. The modulation seen in this curve can be attributed to vibrational modes of DPA. To verify this, we Fourier transformed the time series data. Results of the Fourier transform are presented in the inset of Fig. 2(b). The dashed curves show the spectral feature of the DPA absorp- 


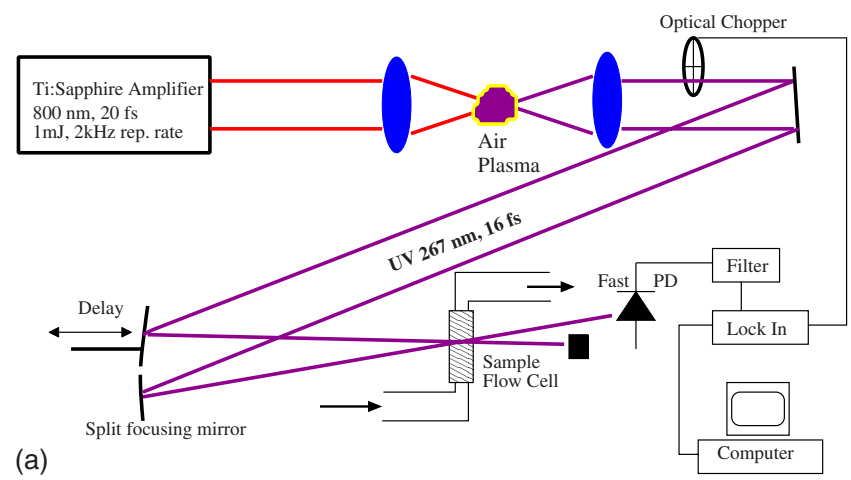

(a)

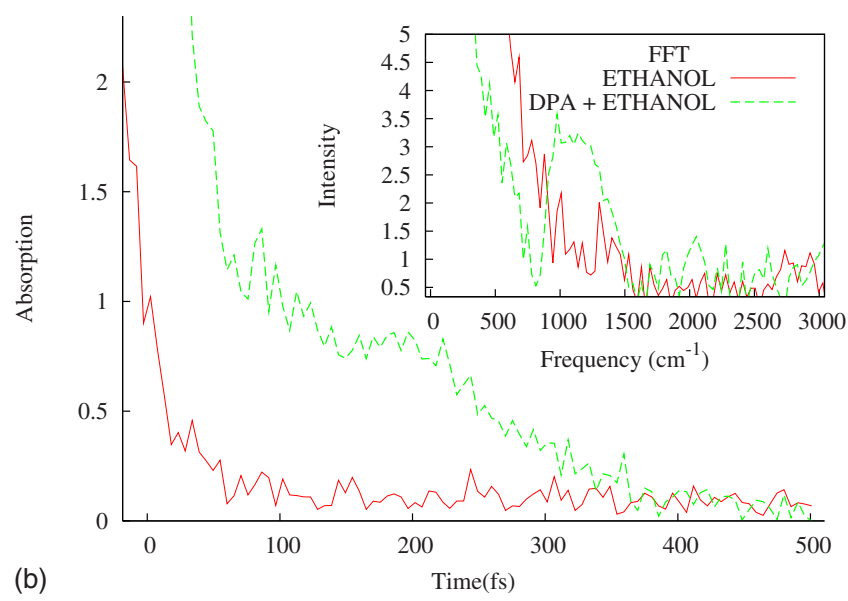

FIG. 2. (Color online) (a) Set up for the uv pump-probe experiment. The output of a Ti:sapphire amplifier is focused in air and generates a plasma. A uv pulse is generated by third harmonic generation and is sent to a split dielectric curved mirror. Half of the mirror is mounted on a delay stage. The absorption of the probe is measured as a function of pump delay with a fast photodiode (PD). The DPA-ethanol solution is circulated using a closed cycle peristaltic pump. (b) Absorption of the probe versus time delay for pure ethanol (solid line) and DPA-ethanol solution (dashed line). The time signal of the DPA curve reveals oscillations which correspond to a frequency range of $1000 \mathrm{~cm}^{-1}$ to $1500 \mathrm{~cm}^{-1}$. The inset shows the Fourier transform of the two time series. The vertical axes are in arbitrary units.

tion induced by the short pump pulses while the solid curves correspond to pure ethanol and are featureless. The frequency range shown in the FFT can be associated with the Raman modes from DPA near 1000, 1400, and $1550 \mathrm{~cm}^{-1}$. The mode structure is not resolved probably due to a low signal to noise ratio. The results indicate that it is possible to observe vibrational modes of DPA with uv impulsive stimulated Raman spectroscopy. The spectral resolution and signal to noise ration can be improved by spatiotemporal optimization of the uv pulses.

\section{MODEL BASED ON THE DENSITY MATRIX}

We present a theoretical model of the pump-probe impulsive technique based on the time dependent density matrix equations which are governed by the Liouville equation. The phenomenological system of equations can be described by the three level energy schematic shown in Fig. 1(b). Here, levels $|a\rangle$ and $|b\rangle$ represent the excited and ground states, respectively, and $|c\rangle$ represents a vibrational level in the ground state. The driving fields, $\Omega_{1}$ and $\Omega_{2}$, are actually the same field in the experiment acting on the levels $|b\rangle$ to $|a\rangle$ and $|c\rangle$ to $|a\rangle$, respectively. by

More specifically, the Hamiltonian of the system is given

$$
H=H_{0}+V \text {, }
$$

where

$$
H_{0}=\sum_{\alpha} \hbar \omega_{\alpha}|\alpha\rangle\left\langle\alpha\left|, \quad V=\sum_{\alpha, \beta} V_{\alpha \beta}\right| \alpha\right\rangle\langle\beta|
$$

For one field having arbitrary polarization, the interaction part of the Hamiltonian is given by

$$
V_{a b}(t)=\wp_{a b} \sum_{i=x, y, z} e_{i}^{a b} E_{i}(t)=\hbar \sum_{i=x, y, z} e_{i}^{a b} \Omega_{i}(t) \equiv \hbar \Omega_{a b}(t) .
$$

The interaction part of the Hamiltonian can be presented as

$$
V_{a b}(t)=\wp_{a b} \sum_{j} \sum_{i=x, y, z} e_{i}^{a b} E_{i}^{\nu_{j}}(t)=\hbar \sum_{j} \Omega_{a b}^{\nu_{j}}(t) .
$$

Our approach is based on the density matrix that allows us to take into account fast relaxation of the medium which is essential. The time evolution of the density matrix is governed by the Liouville-von Neumann equation [20]

$$
\dot{\boldsymbol{\rho}}=-\frac{i}{\hbar}[H, \boldsymbol{\rho}]-\frac{1}{2}\{\Gamma, \boldsymbol{\rho}\},
$$

where the elements are given explicitly as

$$
\begin{gathered}
\dot{\rho}_{a b}=-\Gamma_{a b} \rho_{a b}+i n_{a b} \Omega_{1}-i \rho_{c b} \Omega_{2}, \\
\dot{\rho}_{c a}=-\Gamma_{c a} \rho_{c a}+i n_{c a} \Omega_{2}+i \rho_{c b} \Omega_{1}, \\
\dot{\rho}_{c b}=-\Gamma_{c b} \rho_{c b}+i \rho_{c a} \Omega_{1}-i \rho_{a b} \Omega_{2}, \\
\dot{n}_{b}=i\left(\rho_{b a}-\rho_{a b}\right) \Omega_{1}, \\
\dot{n}_{c}=i\left(\rho_{c a}-\rho_{a c}\right) \Omega_{2}, \\
n_{a}+n_{b}+n_{c}=1,
\end{gathered}
$$

and we have defined $n_{a b}=\rho_{a a}-\rho_{b b}, n_{c a}=\rho_{c c}-\rho_{a a}$, and $\Gamma_{a b}$ $=\gamma_{p h}+i \omega_{a b}, \Gamma_{c a}=\gamma_{p h}-i \omega_{a c}, \Gamma_{c b}=\gamma_{c b}+i \omega_{c b}$.

For simulations, we consider laser fields with Gaussian envelopes

$$
\Omega_{i}=\Omega_{i}^{0} \exp \left[-\left(\frac{t-t_{0}}{\tau}\right)^{2}\right] \cos \left(\omega_{i} t\right), \quad i=1,2,
$$

where $\omega_{i}$ is the frequency of the field, $\tau$ is the duration of the pulse, $t_{0}$ is the pulse center, and $\Omega_{i}^{0}$ is the field amplitude in terms of its Rabi frequency $\wp_{a b} E / \hbar$. There is an additional modification to be made in order to simulate the pump-probe 


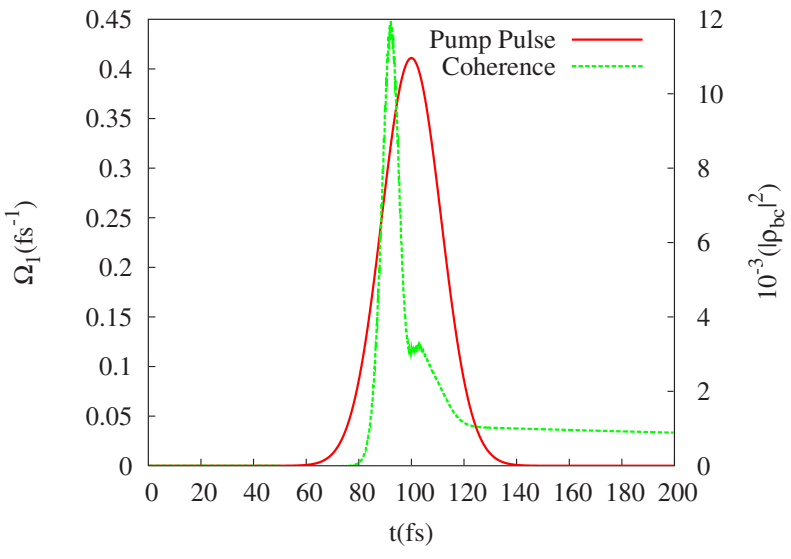

FIG. 3. (Color online) Shown in the figure are the pulse envelope of the uv laser field (solid) and the modulus squared of the coherence $\left|\rho_{b c}\right|^{2}$ (dashed) vs time. The pulse width is $16 \mathrm{fs}$.

technique. We require that a strong pump pulse is applied followed by a weaker delayed probe pulse

$$
\Omega_{\text {pump-probe }}=\Omega_{\text {pump }}(t)+\Omega_{\text {probe }}(t-\Delta t) .
$$

The absorption of the probe pulse will be a function of relative delay $\Delta t$ and carries information about the molecular vibrations. To calculate the absorption of the probe pulse, we use

$$
Q=-\dot{\mathcal{P}} E=\left(\wp_{a b} \dot{\rho}_{b a}+\wp_{a c} \dot{\rho}_{c a}+\text { c.c. }\right) E .
$$

\section{SIMULATIONS}

To gain physical insight into the observed modulation of the probe, we performed numerical simulations. Through simulation, we can determine the optimal parameters of the laser fields which produce impulsive excitation of the molecular media with fast relaxation and estimate their efficiencies. All calculations have been done for parameters corresponding to dipicolinic acid (DPA), but the method is applicable to other biomolecules as well $[25,26]$. The typical values used for simulations are given in the following: $\Omega_{1}^{0}$ $=0.411 \mathrm{GHz}, \Omega_{2}^{0}=\Omega_{1}^{0}, \quad \tau=16 \mathrm{fs}, \quad \omega_{1}=\omega_{2}=2 \pi \times 1.123 \mathrm{PHz}$, $\gamma_{p h}=0.333(1 / \mathrm{fs}), \quad \gamma_{c b}=0.001(1 / \mathrm{fs}), \quad \omega_{a b}=2 \pi \times 1.111 \mathrm{PHz}$, $\omega_{a c}=2 \pi \times 1.081 \mathrm{PHz}$, and $\omega_{c b}=2 \pi \times 0.030 \mathrm{PHz}$. Initial conditions are $\rho_{i j}=0$ for $i \neq j, n_{a}=n_{c}=0$, and $n_{b}=1$.

First, we calculate the excitation of vibrational coherence under the action of a short strong pump pulse. The typical pulse profile and time dependence of the molecular coherence $\rho_{b c}$ are shown in Fig. 3.

The probe pulse is then applied which is delayed with respect to the pump pulse by some period of time $\Delta t$. The absorption of the probe pulse is modulated with the period of the molecular vibration. This modulation is dependent on the width of the pulse used. Shown in Fig. 4(a) is the resulting modulation of the absorption given by Eq. (9) for different values of the pulse duration $\tau$. The dependence of the amplitude of the absorption and the average value of the absorption on the pulse width is useful information in terms of the experiment. First, we consider the average value of the ab-

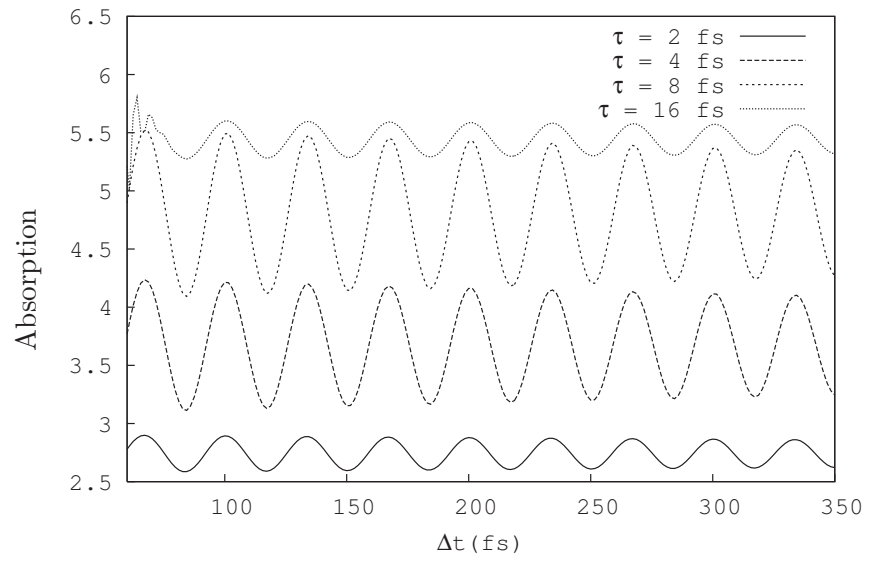

(a)Probe modulation

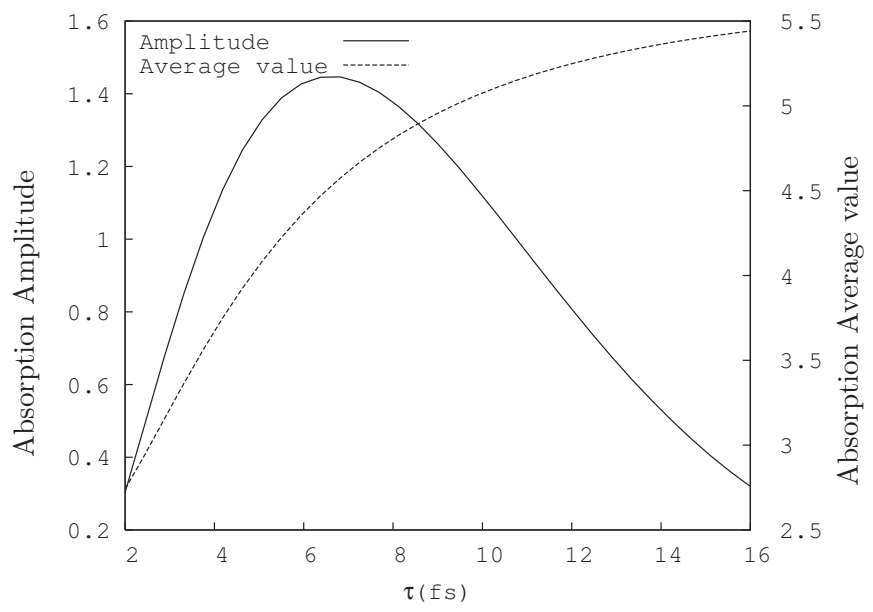

(b)Pulse duration dependence

FIG. 4. (a) Absorption of the probe field vs the relative delay between the pump and probe. The vibrational modulation of the probe is clearly seen. (b) Effect of the pulse duration on the amplitude of the absorption and on the average value of the absorption. The vertical axes are in arbitrary units.

sorption versus pulse width. We note that as the pulse width increases, the average value of absorption does as well. To explain this dependence, we note that the area of the pulse, which is defined by $\theta=\int_{-\infty}^{+\infty} \Omega\left(t^{\prime}\right) / 2 d t^{\prime}$, plays an important role for interaction between the laser field and the atomic system in a two-level atom [19]. For small areas $(\theta \ll \pi)$, the population of the upper state is proportional to the area of the pulse:

$$
n_{a} \simeq \sin \int \Omega\left(t^{\prime}\right) / 2 d t^{\prime}=\sin \theta / 2 \simeq \theta / 2 .
$$

Absorption of the laser pulse increases with the increase of its duration up to a duration that is comparable with the relaxation time for optical coherence. Indeed, for longer times, the optical coherence is given by

$$
\rho_{a b}=-i \int_{0}^{t} d t^{\prime} \Omega\left(t^{\prime}\right) \exp \left[-\Gamma_{a b}\left(t-t^{\prime}\right)\right]
$$



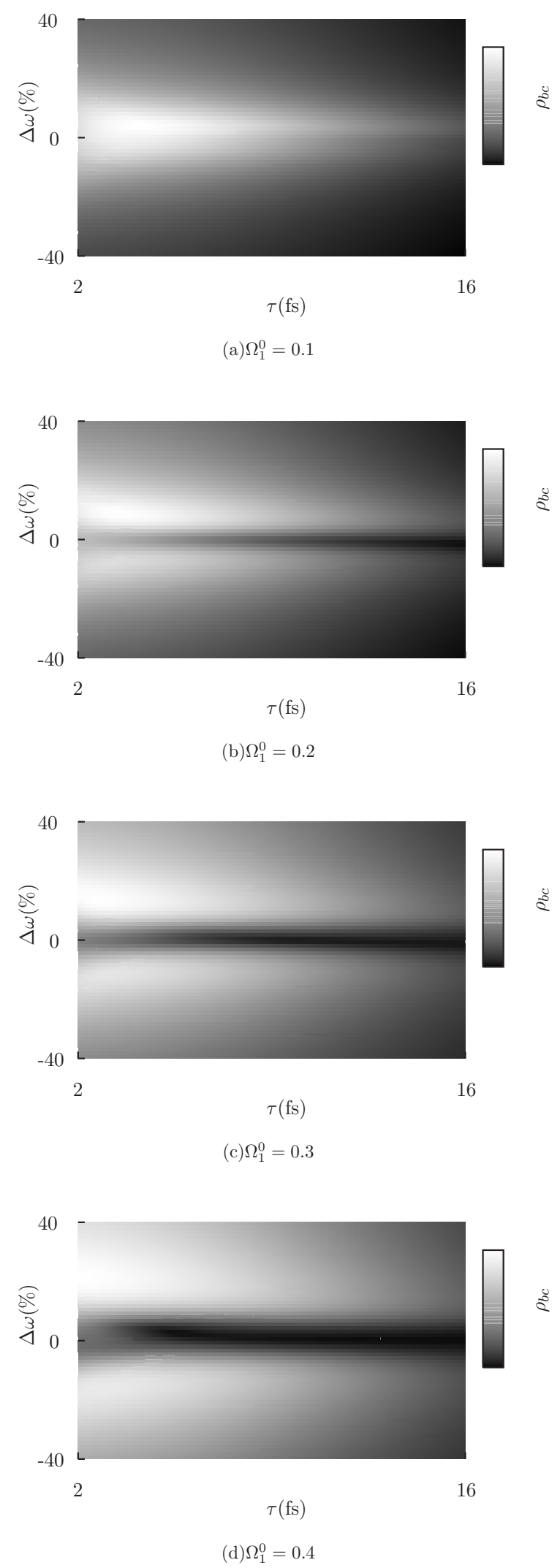

FIG. 5. Amplitude of vibrational coherence vs detuning and pulse width for different field strengths. For weak fields, the coherence has a single resonance. As the field is increased [(a) to (d)] two peaks are formed. The detuning is measured as a percent difference with respect to $\omega_{a b}$. and practically coincides with the steady-state solution. Changing relaxation rates should change the absorption curves.

We have also found that there is an optimal pulse width for the absorption amplitude as is shown in Fig. 4(b). The absorption amplitude is related to the behavior of the excited vibrational coherence $\rho_{a b}$ and $\rho_{a c}$. At short pulses, the spectral width of the laser pulse is big enough to overlap the vibrational frequency, but the area is small, so not much population is transferred to upper level and participating in vibrational coherence excitation. For longer pulses, the area is increased, but the spectral width is not wide enough to excite molecular coherence, so the amplitude of modulation of absorption is going to be smaller. Note that there is practically no modulation of absorption if the pump pulse is longer than 20 fs. We determined that the optimal pulse width for DPA is $\approx 6 \mathrm{fs}$.

Another important pulse parameter is the detuning from one photon resonance. We performed simulations of the vibrational coherence versus one photon detuning and versus pulse duration for different values of field strength $\Omega_{1}^{0}$. The results are summarized in Fig. 5. In the first panel, upper left, the amplitude of the coherence has clear resonance behavior. Thus, we can see that we have two specific parameters, namely, one and two photon resonances. The detuning from one photon resonance produces no impulsive coherence excitation. Several interesting features can be seen in Fig. 5. First, the maximum coherence is shifted toward higher frequencies. It has the simple explanation that there is an optimal detuning. Namely, the detuning should be such that the coupling of laser beam for Stokes and anti-Stokes transition has to be the same.

The second feature is related to dependence of excited coherence as a function of corresponding Rabi frequency. As one can see, for the weaker laser field, the coherence excitation decreases with detuning. But increasing of the laser field actually changes this dependence. Let us note here that, in some sense, the underlying physics is similar to the behavior of a two-level system in intense laser fields at the resonance. Namely, for a strong field, the Rabi frequency becomes comparable to the vibrational frequency. Then, we can describe the interaction between the three-level scheme in the dressed-state basis consisting of the dark and bright states.

Roughly, neglecting the vibrational frequency, the dark and bright states are given by $|D\rangle=(|b\rangle-|c\rangle) / \sqrt{2}$ and $|B\rangle$ $=(|b\rangle+|c\rangle) / \sqrt{2}$, correspondingly. The laser field couples the bright state with an excited electronic state. The population in the bright state is given by $n_{B}=1 / 2 \cos (\theta)$ where $\theta$ is defined as above. Now we see that it is clear that by increasing the laser field, we eventually reach the point where the area of the pulse is $\theta=\pi$, and the population of the bright state leaves unchanged after interaction with the pulse. Because the coherence is related to the population difference between dark and bright states, we can conclude that for this case no coherence is excited. Physically detuning controls coupling between the laser field and the molecular system. Thus, by introducing detuning, we decouple the laser field from the system, and even for the stronger field, we have smaller vibrational coherence excited. But for strong fields, 
we can see that by decreasing coupling via increasing detuning, we first increase vibrational coherence via effectively decreasing Rabi frequency, then, by further increasing detuning, we decrease the vibrational coherence as shown in Fig. 5.

\section{CONCLUSION}

We have experimentally demonstrated a resonant impulsive excitation of DPA molecules dissolved in ethanol. RISRS is a powerful technique which can be used to observe wave packet motion in biomolecules. A full theoretical treatment of the RISRS technique was presented which allows for fast relaxation times. Our simulations provide a means to determine the optimal parameters of the pump laser pulse. The simulations agree with the experimentally observed probe modulation and predict future directions of the experiment.

\section{ACKNOWLEDGMENTS}

We thank Erez Gershgoren, Alexander Goltsov, David Samuels, Petra Sauer, and Raanan Tobey for all their help and useful discussions, and gratefully acknowledge the support from the Office of Naval Research, the Defense Advanced Research Projects Agency, the Robert A. Welch Foundation (Grant No. A-1261), and the National Science Foundation through the Physics Frontier Center and IGERT program.
[1] Norman B. Colthup, Introduction to Infrared and Raman Spectroscopy, 2nd ed. (Academic Press, New York, 1975); Ultrafast Infrared and Raman Spectroscopy, edited by M. D. Fayer (Marcel Dekker, New York, 2001).

[2] R. M. Bowman, M. Dantus, and A. H. Zewail, Chem. Phys. Lett. 161, 297 (1989).

[3] M. Gruebele and A. H. Zewail, J. Chem. Phys. 98, 883 (1993).

[4] A. M. Weiner, D. E. Leaird, G. P. Wiederrecht, and K. A. Nelson, J. Opt. Soc. Am. B 8, 1264 (1991).

[5] R. A. Bartels, S. Backus, M. M. Murnane, and H. C. Kapteyn, Chem. Phys. Lett. 374, 326 (2003).

[6] H. C. Kapteyn, M. M. Murnane, and I. R. Christov, Phys. Today 58, 39 (2005).

[7] S. Backus, J. Peatross, Z. Zeek, A. Rundquist, G. Taft, M. M. Murnane, and H. C. Kapteyn, Opt. Lett. 21, 665 (1996).

[8] J. Chesnoy and A. Mokhtari, Phys. Rev. A 38, 3566 (1988).

[9] H. L. Fragnito, J. Y. Bigot, P. C. Becker, and C. V. Shank, Chem. Phys. Lett. 160, 101 (1989).

[10] E. Gershgoren, J. Vala, R. Kosloff, and S. Ruhman, J. Phys. Chem. A 105, 5081 (2001).

[11] S. Grafe et al., J. Raman Spectrosc. 38, 998 (2007).

[12] M. O. Scully, G. W. Kattawar, P. R. Lucht, T. Opatrny, H. Pilloff, A. Rebane, A. V. Sokolov, and M. S. Zubairy, Proc. Natl. Acad. Sci. U.S.A. 99, 10994 (2002).

[13] D. Pestov, R. K. Murawski, G. O. Ariunbold, Xi Wang, M. Zhi, A. V. Sokolov, V. A. Sautenkov, Y. V. Rostovtsev, A. Dogariu, Y. Huang, and M. O. Scully, Science 316, 265
(2007).

[14] R. Nudelman, B. V. Bronk, and S. Efrima, Appl. Spectrosc. 54, 445 (2000).

[15] S. L. Dexheimer, Q. Wang, L. A. Peteanu, W. T. Pollard, R. A. Mathies, and C. V. Shank, Chem. Phys. Lett. 188, 61 (1992).

[16] U. Banin, A. Bartana, S. Ruhman, and R. Kosloff, J. Chem. Phys. 101, 8461 (1994).

[17] T. Siebert, M. Schmitt, S. Grafe, and V. Engel, J. Raman Spectrosc. 37, 397 (2006).

[18] E. Gershgoren et al., Opt. Lett. 28, 361 (2003).

[19] P. W. Milonni and J. H. Eberly, Lasers (John Wiley \& Sons, New York, 1988).

[20] M. O. Scully and M. S. Zubairy, Quantum Optics (Cambridge University Press, Cambridge, England, 1997).

[21] Z. E. Sariyanni and Y. V. Rostovtsev, J. Mod. Opt. 51, 2637 (2004).

[22] N. A. Anderson, C. G. Durfee, M. M. Murnane, H. C. Kapteyn, and R. J. Sension, Chem. Phys. Lett. 323, 365 (2000).

[23] S. Backus, J. Peatross, M. Murnane, and H. Kapteyn, Opt. Lett. 21, 665 (1996).

[24] C. W. Siders, N. C. T. III, M. C. Downer, A. Babine, A. Stepanov, and A. M. Sergeev, J. Opt. Soc. Am. B 12, 330 (1996).

[25] G. Beadie, Z. E. Sariyanni, Y. V. Rostovtsev, T. Opatrny, J. Reintjes, and M. O. Scully, Opt. Commun. 244, 423 (2005).

[26] D. Pestov et al., Proc. Natl. Acad. Sci. U.S.A. 102, 14976 (2005). 